\title{
Effects of extraction process on phenolic content and antioxidant activity of soybean
}

\author{
Duong Thi Phuong Lien ${ }^{1, *}$, Phan Thi Bich Tram, Ha Thanh Toan ${ }^{2}$ \\ ${ }^{1}$ College of Agriculture and Applied Biology, Can Tho University, Cantho city, Vietnam \\ ${ }^{2}$ Biotechnology Research and Development Institute, Can Tho University, Cantho city, Vietnam
}

Email address:

dtplien@ctu.edu.vn (D. T. P. Lien)

\section{To cite this article:}

Duong Thi Phuong Lien, Phan Thi Bich Tram, Ha Thanh Toan. Effects of Extraction Process on Phenolic Content and Antioxidant Activity of Soybean. Journal of Food and Nutrition Sciences. Special Issue: Food Processing and Food Quality. Vol. 3, No. 1-2, 2015, pp. 33-38. doi: $10.11648 /$ j.jfns.s.2015030102.16

\begin{abstract}
In this research, a set of experiments was carried out for identifying the optimum conditions of independent variables affecting phenolic content extraction efficiency and antioxidant activity of soybean seeds (Glycine max L.). They included the use of different organic solvents (methanol, ethanol and acetone); concentrations of solvent (40,50, 60, 70, 80, and $90 \mathrm{v} / \mathrm{v}, \%)$; the soybean-to-solvent ratio $(1: 4,1: 6 ; 1: 8$ and 1:10) and the number extraction cycles $(2,3$ and 4$)$; the extraction time (2, 3 and 4 hours) and the temperature $\left(30,40,50\right.$ and $\left.60^{\circ} \mathrm{C}\right)$. The extraction abilities of phenolics manifested in forms of total phenolic and total flavonoid contents (TPC and TFC) as well as the antioxidant activity by 1,1-diphenyl-2picrylhydrazyl radical scavenging (DPPH) were used as assessment indicators. Generally, high extraction yield was obtained using aqueous acetone $70 \%$ as solvent; the most suitable soybean-to-solvent ratio was 1:6 for 3 cycles of extraction. The extraction yield could further be increased using a prolonged time of 3 hours at the temperature of $40^{\circ} \mathrm{C}$.
\end{abstract}

Keywords: Soybeans, Polyphenols, Flavonoids, Extraction, Antioxidant Activity, DPPH Radical Scavenging

\section{Introduction}

Soybean (Glycine max L. Merrill) is used extensively for food formulation and it is considered a desirable ingredient for health promotion because of its ability on prevention of cancer, cardiovascular diseases, osteoporosis and menopause symptoms (Adlercreutz and Mazur, 1997; Omoni and Aluko, 2005; Bolanho and Beléia, 2011). These health benefits have been ascribed substantially to the bioactivity of soy polyphenols, including their antioxidant, anti-proliferative, and hypocholesterolemic effects as well as binding to oestrogen receptors (Isanga and Zhang, 2008). The phenolic compounds are known as active antioxidant and as such they are able to scavenge free radicals - important due the deleterious effect of such reactive species, since they are able to oxide bio molecules which may damage cells and cause tissue alterations (Madhavi et al., 1995; Bolanho and Beléia, 2011).

Extraction process is widely known as a separation process of a crude extract of phytochemicals from the raw materials (Chew et al, 2011). Different solvent systems have been used for extraction of polyphenols from plant materials (Chavan et al., 2001). Both extraction yield and extraction activity are strongly dependent on the solvent (Tan et al., 2013). Antioxidative potential of phenolic compounds is strongly affected by the polarity of solvent used in extraction. Hence, the selection of extraction solvents is critical for the complex plant samples. An extraction solvent system is generally selected according to the purpose of extraction, polarity of the interested components, polarity of undesirable components, overall cost, safety and environmental concern (Wang et al., 2008). Aqueous mixtures of acetone, ethanol and methanol have been widely used for extracting phenolic components from botanical materials, especially herbs and medicinal plants (Tabart et al., 2007; Wang et al., 2008). Extraction yield is not only dependent on the solvent but also on the method of extraction (Goli et al., 2004). The extraction method must enable complete extraction of the compounds of interest and must avoid their chemical modification (Zuo et al., 2002). In addition, there is no single universal extraction method applicable for all food matrices because of the complexity of phenolic compounds and its 
interaction with other bioactive compounds presented in the food matrices. There are few factors would contribute in influencing the rate of extraction and quality of extracted bioactive phenolic compounds, including method of extraction, solvent type, solvent concentration, contact time, extraction temperature, solid to solvent ratio and particle size (Chew et al., 2011; Jin Dai et al., 2010; Pinelo et al., 2005).

According to Silva et al. (2007), the extraction method of phenolic compounds differs from plant to plant and an ideal extraction method for a particular phenolic source has to be individually designed and optimized. The objective of this study was to evaluate the efficacy of solvent types, solvent concentrations, the soybean-to-solvent ratios as well as the number extraction cycles, the extraction temperature and time for the extraction of phenolic contents and antioxidant activity of soybean.

\section{Materials and Methods}

Preparation of samples: Soybeans (Glycine max L., МТÐ 760 variety) were supplied from Department of Agricultural Genetic, College of Agricultural and Applied Biology, Cantho University. The cleaned soybeans were ground (Giao et al., 2009), defatted in a Soxhlet extractor with hexane for 10h (Weidner et al., 2012) and then were stored at $5^{\circ} \mathrm{C}$ after removal of hexane.

Experimental design for the extraction of TPC and TFC from soybean: A mass of $0.5 \mathrm{~g}$ of defatted soybean powder was extracted twice with $5 \mathrm{ml}$ solvent with shaking and the two extracts were combined for determination the TPC, TFC and antioxidant potential.

Studying effects of solvent types on TPC, TFC and antioxidant activity was carried out with three types of solvents as methanol, acetone and ethanol $(70 \%, \mathrm{v} / \mathrm{v})$. Studying effects of solvent concentrations on TPC, TFC and antioxidant activity was carried out with the concentration of solvent as 40, 50, 60, 70, 80 and $90(\%, v / v)$. To study the effects of soybean-to-solvent ratio $(1: 4,1: 6,1: 8$ and 1:10) and the number extraction cycles (2, 3 and 4$)$ on TPC, TFC and antioxidant activity was designed in form two factor experiment. Similarly, the effects of extraction time (2, 3 and 4 hours) and temperature $\left(30,40,50\right.$ and $\left.60^{\circ} \mathrm{C}\right)$ on TPC, TFC and antioxidant activity was investigated by two factor design.

Determination of the TPC in the extracts: The TPC was estimated by Folin-Ciocalteu method (Susu Jiang et al., 2013). The total phenolic content of samples was expressed as milligrams gallic acid equivalents per gram of dry matter (mg GAE/g).

Total flavonoid content (TFC) The TFC was determined by the colorimetric method as described by Ozsoy et al. (2008). The results were expressed as milligrams of quercetin equivalents (QE) per $\mathrm{g}$ of dry matter sample (mg QE/g).

Antioxidant activity: Antioxidant activity of the phytochemicals extracted from soybean was assessed by measuring their radical scavenging activity that was measured by the bleaching of the purple-coloured methanol solution of 1,1-diphenyl-2-picrylhydrazyl radical (DPPH). This spectrophotometric assay uses stable DPPH radical as a reagent and was described by Anshu et al. (2011).

Statistical analysis: All the experimental results were analysed using Portable Statgraphics Centurion software (Version 15.2.11.0). Every assay was done in triplicates. Analysis of variance (ANOVA) with LSD test was used to determine the significant differences $(p<0.05)$ between means.

\section{Results and Discussions}

\subsection{Effects of Types of Solvent on the Contents of TPC, TFC and Antioxidant Activity}

Figures 1 show the TPC and TFC results of soybean extracted from three types of solvents as acetone, methanol and ethanol. Most efficient extractor of polyphenols (TPC and TFC) among the examined solvents seemed to be acetone solvent. The result is suitable to their ability to deactivate stabile DPPH radicals has been shown in Figure 2. The strongest properties to DPPH radicals showed acetone extracts (\% scavenging radicals at the level of 76.4). Many studies showed that acetone is the best solvent for proanthocyanidins and tannins extraction (Chirinos et al., 2007; Tabart et al., 2007). These facts are in accordance with polarity of the solvent used for the extraction and solubility of phenolic compounds in them. It is interesting to note that the polarity of acetone, ethanol and methanol is $0.355,0.654$ and 0.762 , respectively (Tan et al., 2013). Aqueous acetone is a good solvent for polar antioxidants and more useful for extracting.
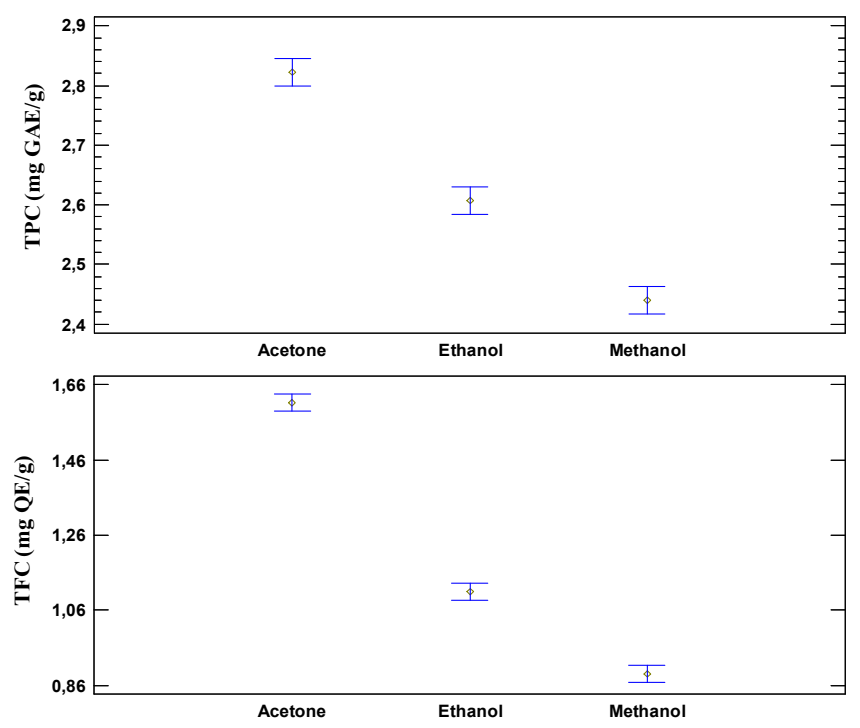

Figure 1. TPC and TFC contents from soybean extracted by various solvents (means and 95\% LSD intervals).

Polyphenols from protein matrices, since they appear to degrade the polyphenol-protein complexes (Chirinos et al., 2007; Al-Farsi and Lee, 2008). In fact, the use of aqueous acetone has several advantages to the use of aqueous ethanol 
and aqueous methanol, for example higher extraction efficiency, suggesting the use of aqueous acetone as extraction solvent for the following stages in this study. However, there is still a need to check if using different water percentages in acetone $(\%, v / v)$ is possible to increase the extraction efficiency of TPC from soybean in the experiment.

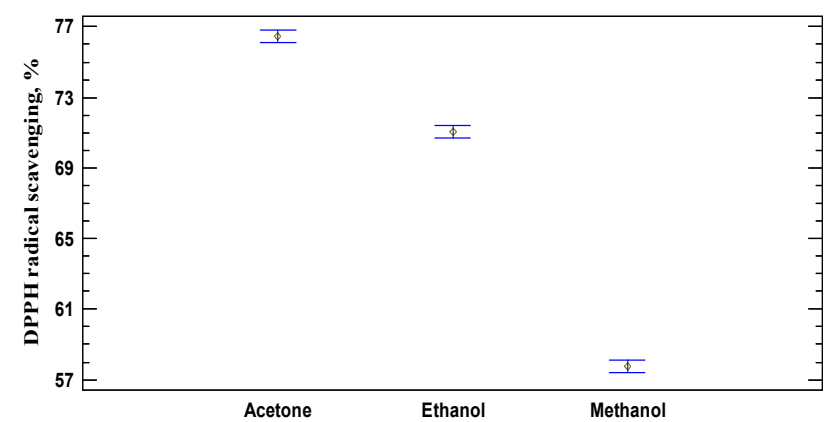

Figure 2. Antioxidant activities from soybean extracted by various solvents (means and 95\% LSD intervals).

\subsection{Effects of Acetone Concentration on the TPC, TFC Contents and Antioxidant Activity}

The effects of acetone concentration in the extraction solvent on the content of polyphenols and flavonoids in soybean extracts were shown in Figure 3. The correspondently soybean antioxidant activities was expressed on Figure 4. The acetone concentration had a significant effect $(p<0.05)$ on the extraction efficiency of both TPC and TFC from soybean.
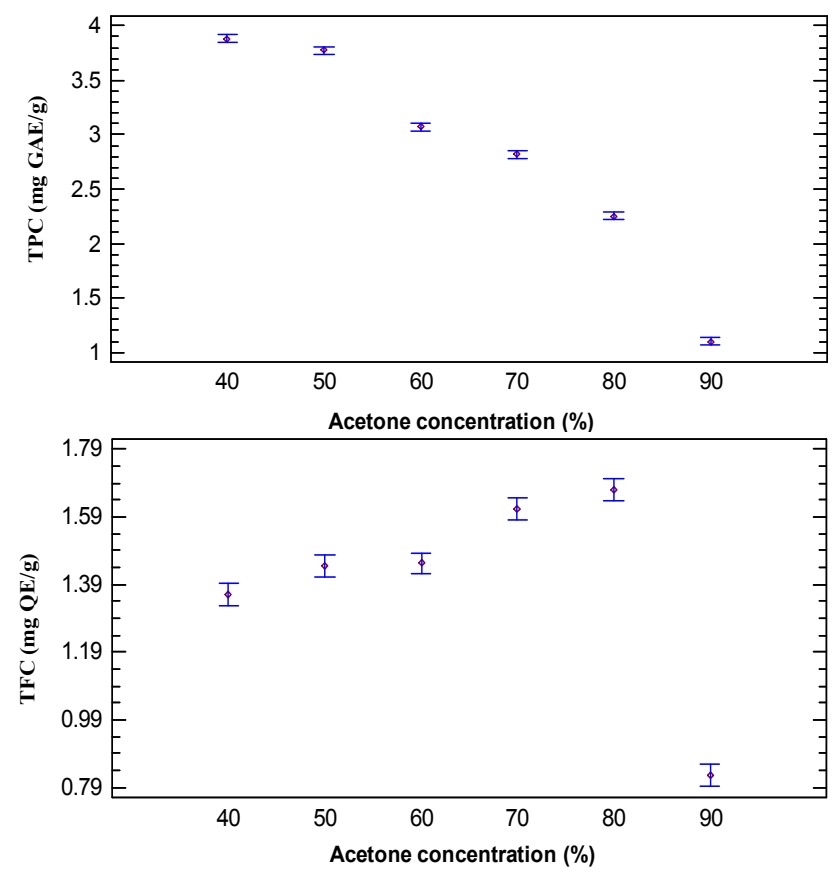

Figure 3. TPC and TFC contents from soybean extracted by various concentrations of acetone (means and 95\% LSD intervals)

As can be seen from Figure 3, the TPC content increases as decreasing the acetone concentration. However, the TFC content follows a curve shape and TFC of soybean extracts reached a maximum at $70-80 \%$ acetone $(\mathrm{v} / \mathrm{v})$ followed by a significant $(p<0.05)$ decrease with further increase in the concentration of the acetone in the extraction medium. The change in TFC content was same to the change in antioxidant activity (Figure 4) that reached to maximum value at $70 \%$ acetone. The different rule in the change of TPC might be due to the more water in aqueous acetone solution is the more water soluble proteins extracted. The reaction of these soluble proteins and Folin reagent resulted in the change of absorbance reading. Form all observations, a moderately polar solvent of $70 \%$ acetone $(\mathrm{v} / \mathrm{v})$ was chosen for the next step of study.

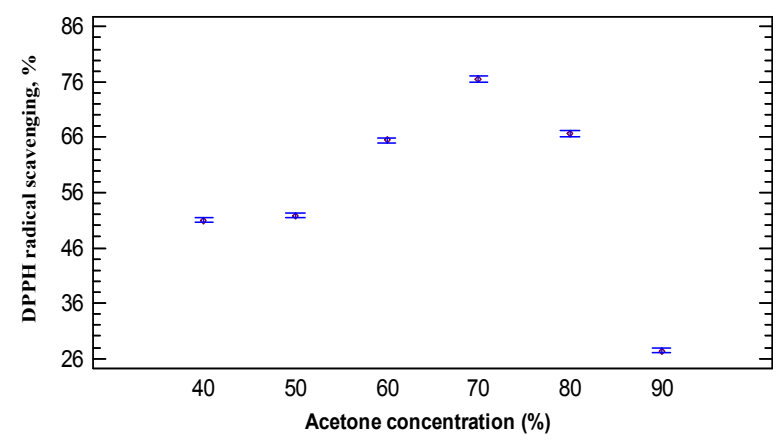

Figure 4. Antioxidant activities from soybean extracted by various concentrations of acetone (means and 95\% LSD intervals).

\subsection{Effects of the Soybean-to-Solvent Ratio and the Number Extraction Cycles on the TPC and TFC Contents}

The effects of soybean-to-solvent ratio and number extraction cycles on total phenolic content (TPC) and total flavonoid content (TFC) are shown in can be observed that both the number extraction cycles and the soybean-tosolvent ratio effect significantly on TPC and TFC contents. Soybean-to-solvent ratio of 1:6 (w/v) showed high amount of TPC and TFC. A further increase in soybean-to-solvent ratio from 1:6 to $1: 10$ did not significantly $(\mathrm{p}>0.05)$ increase both values of TPC and TFC. According Tan et al. (2011), a high solid to solvent ratio was found to be favorable in extraction of phenolic compounds. These results were consistent with mass transfer principles where the driving force for mass transfer is considered to be the concentration gradient between the solid and the solvent. A high solid-tosolvent ratio could promote an increasing concentration gradient, resulting in an increase of diffusion rate that allows greater extraction of solids by solvent (Cacace and Mazza, 2003; Al-Farsi and Chang, 2007). In addition, the chance of bio-active components coming into contact with extracting solvent expanded with increase amount of extraction solvent, leading to higher leaching out rates (Zhang et al., 2007). However, active component yields will not continue to increase once equilibrium is reached (Herodež et al., 2003).

Multiple-step extraction is an important method to improve the extraction yield of polyphenols (Chen et al., 2013). Three cycles extraction showed higher yield of both TPC and TFC than that of using two cycles of extraction. 
However, four cycles of extraction could not improve the yield of both TPC and TFC (Fig. 5).
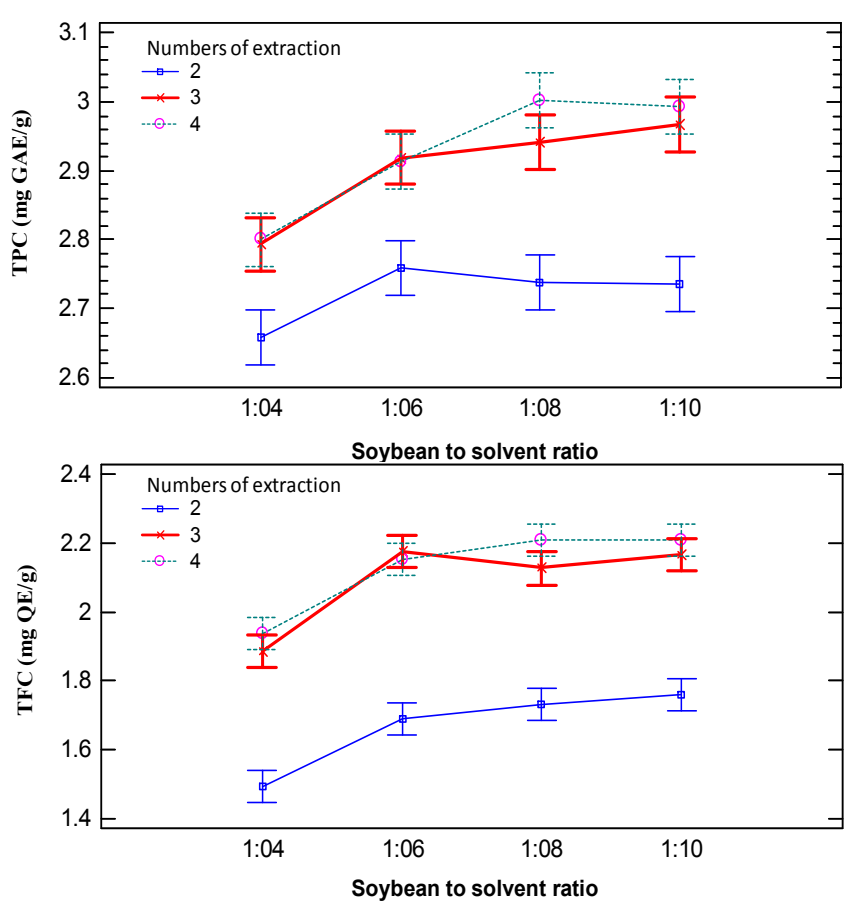

Figure 5. Effects of soybean-to-solvent ratio and number extraction cycles on TPC and TFC of soybean (means and 95\% LSD intervals)

From obtained results, the soybean-to-solvent ratio of 1:6 $(\mathrm{w} / \mathrm{v})$ and three cycles extraction are optimum conditions for extraction TPC and TFC from soybean.

\subsection{Effects of the Extraction time and Temperature on the Contents of TPC, TFC and Antioxidant Activity}
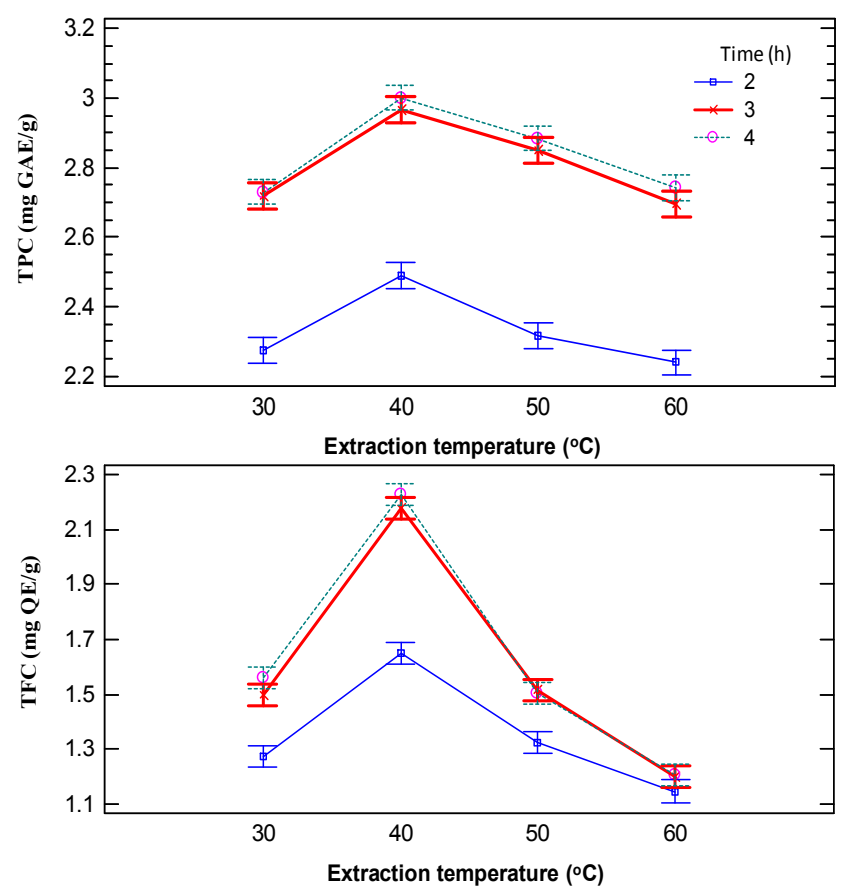

Figure 6. Effects of extraction time and temperature on TPC and TFC contents of soybean (means and $95 \%$ LSD intervals)

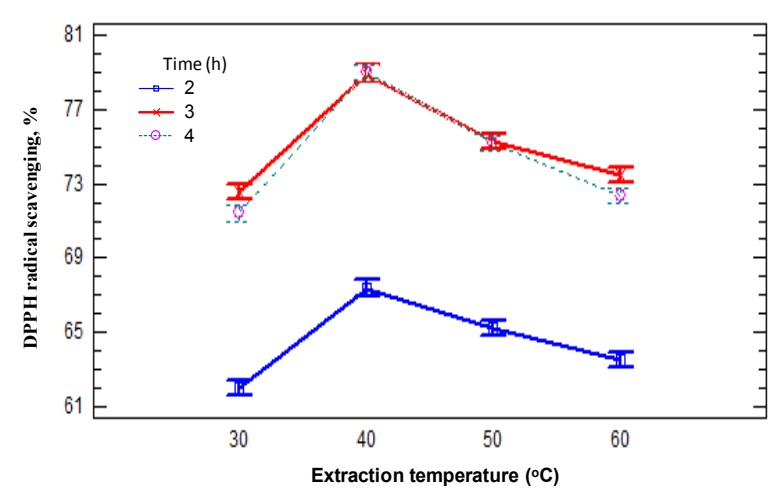

Figure 7. Effects of extraction time and temperature on antioxidant activity of soybean (means and 95\% LSD intervals)

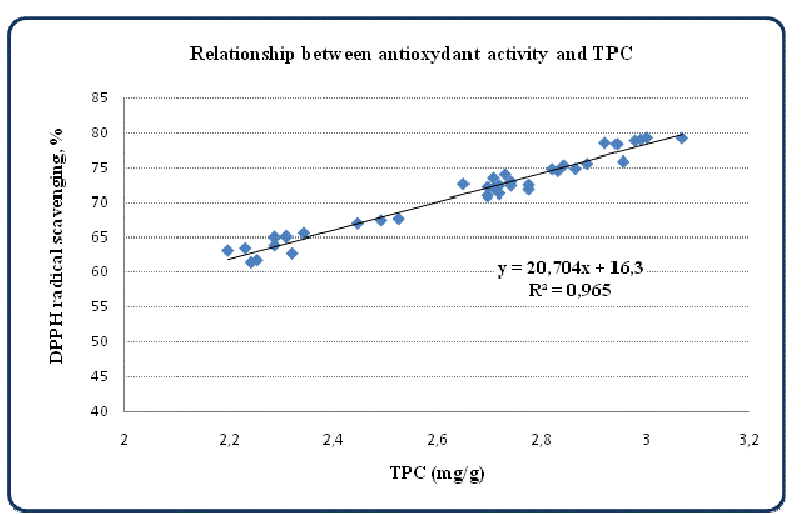

Figure 8. Correlation between DPPH free radical scavenging and TPC content

The selection of appropriate extraction time and temperature was the final step in experiments. The changes in TPC and TFC contents by extraction time and temperature were described in Figure 6. The effect of these factors on antioxidant activity was shown in Figure 7. The TPC and TFC contents increased significantly with increasing temperature reaching a peak at $40^{\circ} \mathrm{C}$, and then decreased significantly.

According Spigno et al. (2007), extraction temperature impacts the solubility, mass-transfer rate, and stability of phenolic compounds. The results confirmed the fact that, below a certain limit, higher temperatures improve the efficiency of extraction due to enhancement of the diffusion rate and the solubility of analytes in solvents (Ju and Howard, 2003). Beyond the limit, high extraction temperatures decrease TPC and TFC contents. It has been observed in the study that with the prolongation of the extraction time from 2 to 3 hours the output of polyphenols and flavonoids in solvent increased significantly. But there is no significant difference in TPC and TFC contents with the prolongation of extraction time up to 4 hours. These phenomena could be well explained by the Fick's second law of diffusion, predicting that a final equilibrium between the solute concentrations in the solid matrix and in the solvent might be reached after a certain time (Silva et al., 2007). Effects of time and temperature on DPPH free-radical scavenging potential showed a similar trend of TPC and TFC. Interesting result was the fine linear correlation between DPPH free 
radical scavenging and TPC content with $\mathrm{R}^{2}=0.97$ (Figure 8), confirming that polyphenols are likely to contribute to the radical scavenging activity of these plant extracts (Miliauskas et al., 2004). Similar results have also been reported for different plants by various studies (Katalinic' et al., 2004; Maksimovic' et al., 2005; Miliauskas et al., 2004; Yu et al., 2005 and Turkmen et al., 2006).

\section{Conclusions}

Acetone was found to be the best solvent for the soybean extraction. Subsequently, the optimum conditions for maximum TPC, TFC and antioxidant activity were found to be acetone concentration $70 \%(\mathrm{v} / \mathrm{v})$, soybean-to-solvent ratio of 1:6 (w/v), three cycles extraction, 3 hours for each cycle extraction at the temperature $40^{\circ} \mathrm{C}$. Operating with these conditions, the TPC and TFC contents were $2.97 \pm$ $0.041 \mathrm{mgGAE} / \mathrm{g}(\mathrm{db})$ and $2.18 \pm 0.057 \mathrm{mgQE} / \mathrm{g}(\mathrm{db})$, respectively. The DPPH free radical scavenging reaches $78,96 \pm 0,34 \%$ and has a good correlation to the total polyphenol content.

\section{References}

[1] Adlercreutz H. and Mazur W. 1997. Phyto-oestrogens and western diseases. Ann. Med, v. 29, p. 95-102.

[2] Al-Farsi, M. A. and Chang, Y. L. 2007. Optimization of phenolics and dietary fiber extraction from date seeds. Food Chemistry 108: 977-985.

[3] Al-Farsi, M. A. and Lee, C. Y. 2008. Optimization of phenolics and dietary fiber extraction from date seeds. Food Chemistry 108: 977-985.

[4] Anshu Singh, Arindam Kuila, Geetanjali Yadav and Rintu Banerjee. 2011. Process Optimization for the Extraction of Polyphenols from Okara. Food Technol. Biotechnol. 49 (3) 322-328. ISSN 1330-9862

[5] Bolanho Beatriz Cervejeira, Adelaide Del Pino Beléia. 2011. Bioactive compounds and antioxidant potential of soy products. Alim. Nutr., Araraquara, v. 22, n. 4, p. 539-546, out./dez. 2011. ISSN 0103-4235, ISSN 2179-4448 on line.

[6] Cacace, J. E. and Mazza, G. 2003. Mass transfer process during extraction of phenolic compounds from milled berries. Journal of Food Engineering 59: 379-389.

[7] Chavan, U. D., Shahidi, F. and Naczk, M. 2001. Extraction of condensed tannins from beach pea (Lathyrus maritimus L.) as affected by different solvents. Food Chemistry 75: 509-512.

[8] Chen Xiao-xin, Xiao-bing Wu, Wei-ming Chai, Hui-ling Feng, Yan Shi, Han-tao Zhou, Qing-xi Chen. 2013. Optimization of extraction of phenolics from leaves of Ficus irens. Journal of Zhejiang University-SCIENCE B (Biomedicine \& Biotechnology), 903-915. ISSN 1673-1581 (Print); ISSN 1862-1783 (Online), www.zju.edu.cn/jzus; www.springerlink.com

[9] Chew, K.K., Ng, S.Y., Thoo, Y.Y., Khoo, M.Z., Wan Aida, W.M. and Ho, C.W. (2011). Effect of ethanol concentration, extraction time and extraction temperature on the recovery of phenolic compounds and antioxidant capacity of Orthosiphon stamineus extracts. International Food Research Journal, 18: $1427-1435$.

[10] Chirinos, R., Rogez, H., Campos, D., Pedreschi, R. and Larondelle, Y. 2007. Optimization of extraction conditions of antioxidant phenolic compounds from mashua (Tropaeolum tuberosum Ruíz \& Pavón) tubers. Separation and Purification Technology 55(2): 217-225.

[11] Giao M.S., Pereira C.I., Fonseca S.C., Pintado M.E., Malcata F.X. 2009. Effect of particle size upon the extent of extraction of antioxidant power from the plants Agrimonia eupatoria, Salvia sp. and Satureja montana. Food Chem. 117, 412-416.

[12] Goli, A. H., Barzegar, M. and Sahari, M. A. 2004. Antioxidant activity and total phenolic compounds of pistachio (Pistachia vera) hull extracts. Food Chemistry 92:521-525.

[13] Herodež, Š. S., Hadolin, M., Škerget, M. and Knez, Ž. 2003. Solvent extraction study of antioxidants from Melissa officinalis L. leaves. Food Chemistry 80: 275-282

[14] Isanga, J., \& Zhang, G. (2008). Soybean bioactive components and their implications to health -a review. Food Reviews International, 24, 252-276.

[15] Jin Dai 1, 2 and Russell J. Mumper. 2010. Plant Phenolics: Extraction, Analysis and Their Antioxidant and Anticancer Properties. Molecules 15, 7313-7352; doi:10.3390/molecules15107313. ISSN 1420-3049, www.mdpi.com/journal/molecules

[16] Ju ZY, Howard LR. 2003. Effects of solvent and temperature on pressurized liquid extraction of anthocyanins and total phenolics from dried red grape skin. J Agric Food Chem. 51(18):5207-5213.

[17] Katalinic', V., Milos, M., Modun, D., Music', I., \& Boban, M. (2004). Antioxidant effectiveness of selected wines in comparison with (+)- catechin. Food Chemistry, 80, 593-600.

[18] Madhavi D. L.; Deshpande S. S. and Salunkhe D. K. 1995. Food antioxidants: technological, toxicological and health perspectives. New York: Marcel Dekker, 490p.

[19] Maksimovic', Z., Malenc`ic', D., \& Kovac`evic', N. (2005). Polyphenol contents and antioxidant activity of Maydis stigma extracts. Bioresource Technology, 96, 873-877.

[20] Miliauskas, G., Venskutonis, P. R., \& van Beek, T. A. (2004). Screening of radical scavenging activity of some medicinal and aromatic plant extracts. Food Chemistry, 85, 231-237.

[21] Nihal Turkmen, Ferda Sari, Y. Sedat Velioglu. 2006. Effects of extraction solvents on concentration and antioxidant activity of black and black mate tea polyphenols determined by ferrous tartrate and Folin-Ciocalteu methods. Food Chemistry $99,835-841$.

[22] Omoni, A. O., \& Aluko, R. E. (2005). Soybean foods and their benefits: Potential mechanisms of action. Nutrition Reviews, 63, 272-283.

[23] Ozsoy, N., Can, A., Yanardag, R. \& Akev, N. 2008. Antioxidant activity of Smilax excelsa L. leaf extracts. Food Chemistry 110: 571-583.

[24] Pinelo, M., Rubilar, M., Jerez, M., Sineiro, J. and Nuñez, M. J. 2005. Effect of solvent, temperature, solvent to solid ratio on the total phenolic content and antiradical activity of extracts from different components of grape pomace. Journal of Agricultural and Food Chemistry 53: 2111-2117 
[25] Silva, E. M., Rogez, H. and Larondelle, Y. 2007. Optimization of extraction of phenolics from Inga edulis leaves using response surface methodology. Separation and Purification Technology 55: 381-387.

[26] Spigno, G., Tramelli, L. and De Faveri, D. M. 2007. Effects of extraction time, temperature and solvent on concentration and antioxidant activity of grape marc phenolics. Journal of Food Engineering 81:200-208.

[27] Susu Jiang, Weixi Cai and Baojun Xu (2013) Food Quality Improvement of Soy Milk Made from Short-Time Germinated Soybeans. Foods 2, 198-212.

[28] Tabart, J., Kevers, C., Sipel, A., Pincemail, J., Defraigne, J. O. and Dommes, J. 2007. Optimisation of extraction of phenolics and antioxidants from black currant leaves and buds and of stability during storage. Food Chemistry 105: 1268-1275.

[29] Tan M. C., Tan C. P. and Ho C. W. 2013. Effects of extraction solvent system, time and temperature on total phenolic content of henna (Lawsonia inermis) stems. International Food Research Journal 20(6): 3117-3123.

[30] Tan P. W., Tan C. P. and Ho C. W. 2011. Antioxidant properties: Effects of solid-to-solvent ratio on antioxidant compounds and capacities of Pegaga (Centella asiatica). International Food Research Journal 18: 557-562.
[31] Wang, J., Sun, B. G., Cao, Y., Tian, Y. and Li, X. H. 2008. Optimization of ultrasound-assisted extraction of phenolic compounds from wheat bran. Food Chemistry 106: 804-810.

[32] Weidner, S.; Powałka, A.; Karamać, M.; Amarowicz, R. 2012. Extracts of phenolic compounds from seeds of three wild grapevines-Comparison of their antioxidant activities and the content of phenolic compounds. Int. J. Mol. Sci. 13, 34443457 .

[33] Yu J., Ahmedna M., \& Goktepe I. (2005). Effects of processing methods and extraction solvents on concentration and antioxidant activity of peanut skin phenolics. Food Chemistry, 90, 199-206.

[34] Zhang, S. Q., Bi, H. M. and Liu, C. J. 2007. Extraction of bioactive components from Rhodiola sachalinensis under ultrahigh hydrostatic pressure. Separation and Purification Technology 57: 277-282.

[35] Zuo, Y., Chen, H. and Deng, Y. 2002. Simultaneous determination of catechins, caffeine and gallic acids in green, oolong, black and pureh teas using HPLC with a photodiode array detector. Talanta 57: 307-316. 